\title{
On the uncertainty of defocus methods for 3D particle tracking velocimetry
}

\author{
C. Cierpka ${ }^{1 *}$, R. Barnkob ${ }^{2}$, S. Sachs ${ }^{1}$, M. Chen ${ }^{3}$, P. Mäder ${ }^{3}$, M. Rossi ${ }^{4}$
}

\author{
${ }^{1}$ Institute of Thermodynamics and Fluid Mechanics, Technische Universität Ilmenau, P.O. Box 100565, \\ D-98684 Ilmenau, Germany \\ ${ }^{2}$ Heinz-Nixdorf-Chair of Biomedical Electronics, Department of Electrical and Computer Engineering, \\ Technical University of Munich, TranslaTUM, 81675 Munich, Germany \\ ${ }^{3}$ Group for Software Engineering for Safety-Critical Systems, Technische Universität Ilmenau, P.O. Box \\ 100565, D-98684 Ilmenau, Germany \\ ${ }^{4}$ Department of Physics, Technical University of Denmark, DTU Physics Building 309, DK-2800 Kongens \\ Lyngby, Denmark \\ * christian.cierpka@tu-ilmenau.de
}

Defocus methods have become more and more popular for the estimation of the 3D position of particles in flows (Cierpka and Kähler, 2011; Rossi and Kähler, 2014). Typically the depth positions of particles are determined by the defocused particle images using image processing algorithms. As these methods allow the determination of all components of the velocity vector in a volume using only a single optical access and a single camera, they are often used in, but not limited to microfluidics. Since almost no additional equipment is necessary they are low-cost methods that are meanwhile widely applied in different fields. To overcome the ambiguity of perfect optical systems, often a cylindrical lens is introduced in the optical system which enhances the differences of the obtained particle images for different depth positions. However, various methods are emerging and it is difficult for non-experienced users to judge what method might be best suited for a given experimental setup. Therefore, the aim of the presentation is a thorough evaluation of the performance of general advanced methods, including also recently presented neural networks (Franchini and Krevor, 2020, König et al., 2020) based on typical images.

For the assessment of the uncertainty and the tracking probability in different imaging situations, the ground truth has to be known. For this reason, synthetic images were used which show a systematic variation of the imaging conditions, in this case, the degree of astigmatism (none, mild, strong), the noise level and the number of particle images per pixel (Rossi, 2020). In addition experimental images were obtained using a typical defocus setup $(M=20)$, including a cylindrical lens $\left(f_{c}=250 \mathrm{~mm}\right)$. For further details the reader is refereed to Barnkob et al. (2021) and may access the data soon ${ }^{1}$.

Three different methods to determine the depth position by evaluating certain features of the defocused particle images are compared. If a model function (MF) is known, the width and height of the particle images can be evaluated with classical image analysis and related to the depth position (Cierpka et al., 2010). However, often such a model function is unknown or too complex. In this case the cross-correlation (CC) with a template image that is determined from a set of calibration images can be used (Barnkob et al. 2015; Rossi and Barnkob, 2020). A high value of the correlation between template and particle image indicates the same depth position. Another recently presented method is the use of image recognition by modern machine learning neural networks (NN) to determine the volumetric particle position (Franchini and Krevor, 2020, König et al., 2020). The image aberrations serve as features in this case and the network can be trained to relate these features to a corresponding depth position.

The uncertainty and the recall (i.e. the ratio between valid detected particles and total number of particles in the image) were evaluated to compare the different methods using synthetic and experimental images with different noise levels and particle image overlapping. In general, it can be stated that all three methods are able to determine the 3D position of particles. The MF algorithms require astigmatic aberrations of the images and show low uncertainties especially for mild astigmatism and low particle image concentration. As a model function (in this case a Gaussian intensity distribution) was used, no image pre-processing had to be applied. CC methods worked for all cases and especially well in the case of large image overlap and noise

1 https://defocustracking.com/datasets/ 
levels. However, special care has to be given when the particle images differ strongly in different positions of the field of view. For both approaches, CC and MF the error in the depth direction never exceeded 3\% of the whole measurement depth for typical experimental conditions. In the current study NN algorithms were not able to reach similar uncertainties as compared to the other techniques. Especially as the synthetic data consists of the same intensity distribution per particle image shifted to different positions within the field of view, the amount of training data is too limited. If experimental images are used the algorithms work considerably better (König et al. 2020).

In the final presentation, the performance of the different algorithms will be shown. The reasons for the observed differences will be discussed together with guidelines for which algorithm may be used in which setup.

\section{Acknowledgements}

CC and SS acknowledge financial support by the DFG under Grant No. CI 185/8-1 within the PP 2045 MehrDim- Part. MR acknowledges financial support by the Villum Foundation under the Grant No. 00022951. PM and CC acknowledge financial support by the Carl Zeiss Foundation's Grant: DeepTurb. The authors also want to thank Dr Thomas Fuchs (UniBW Munich) for implementing the edge-detection algorithm.

\section{References}

Barnkob R, Cierpka C, Chen M, Sachs S, Mäder P, and Rossi M (2021) Optimization of defocus particle tracking based on image recognition. Measurements Science and Technology 32:094011

Barnkob R, Kähler CJ, and Rossi M (2015) General defocusing particle tracking. Lab on a Chip 15:35563560

Cierpka C and Kähler CJ (2011) Particle imaging techniques for volumetric three-component (3d3c) velocity measurements in microfluidics. Journal of Visualization 15:1-31

Cierpka C, Rossi M, Segura R, and Kähler CJ (2010) On the calibration of astigmatism particle tracking velocimetry for microflows. Measurement Science and Technology 22:015401

Franchini S and Krevor S (2020) Cut, overlap and locate: a deep learning approach for the 3d localization of particles in astigmatic optical setups. Experiments in Fluids 61:140

König J, Chen M, Rösing W, Boho D, Mäder P, and Cierpka C (2020) On the use of a cascaded convolutional neural network for three-dimensional flow measurements using astigmatic PTV. Measurement Science and Technology 31:074015

Rossi M (2020) Synthetic image generator for defocusing and astigmatic PIV/PTV. Measurement Science and Technology 31:017003

Rossi M and Barnkob R (2020) A fast and robust algorithm for general defocusing particle tracking. Measurement Science and Technology 32:014001

Rossi M and Kähler CJ (2014) Optimization of astigmatic particle tracking velocimeters. Experiments in Fluids 55 